\title{
A Class of Weibull Mixtured Distributions
}

\section{Mian Arif Shams Adnan* and Humayun Kiser}

Department Of Statistics, Jahangirnagar University, Savar, Dhaka 1342, Bangladesh

\begin{abstract}
We have derived a class of mixture distributions which we call weibull mixtures of distributions. Estimation of unknown parameters along with some properties of these distributions are also prescribed.
\end{abstract}

Keywords: Mixing distribution; Mixtured distribution; Weibull distribution

\section{Introduction}

Mixture distribution was first coined in 1894. A number of authors like Pearson, Rider, Blichke, Chahine, Roy, et al., authors [1-13] defined mixtures of two distributions and studied various mixtured distributions which they called poisson mixture, binomial mixture, negative binomial mixture, Chi-square mixture, Erlang mixture, Laplace mixture, Rayleigh mixture, $F$, Dual mixture of distributions. Weibull distribution is widely being used in bio-statistics, but weibull mixture distribution has not yet been premeditated. In the present paper, we define first the weibull mixture of distributions and then weibull mixtures of normal, lognormal, gamma, exponential, beta, rectangular, erlang, chi-square, $\mathrm{t}$ and $\mathrm{F}$ distributions and studied some of their properties.

\section{Preliminaries}

A mixture distribution is a weighted average of probability distribution of positive weights that sum to one. The distributions thus mixed are called the components of the mixture. The weights themselves comprise a probability distribution called the mixing distribution. Because of this property of weights, a mixture is in particular again a probability distribution. Mixtures occur most commonly when the parameter $\theta$ of a family of distributions, given by the density by the density function $f(x, \theta)$, is itself subject to the change variation. The mixing distribution $g(x ; \theta)$ is then a probability distribution on the parameter of the distributions. The general formula for the finite mixture is $\sum_{i=1}^{k} f\left(x ; \theta_{i}\right) g\left(\theta_{i}\right)$; the infinite analogue, in which $g$ is a density function, is $\int f(x ; \theta) g(\theta) d \theta$.

\section{Main Results}

Here in this paper, we define the weibull mixtures of some well known distributions such as normal, lognormal, gamma, exponential, beta, rectangular, erlang, chi-square, $\mathrm{t}$ and $\mathrm{F}$ distributions. Then some characteristics of these distributions such as characteristic functions, moments, and shape chaacteristics are also obtained. The main results of the paper are presented in form of definitions and theorems. Comparison of the probability density functions and the first two moments are prescribed in the tertiary section.

\section{Definition 3.1}

A random variable $\mathrm{X}$ is said to have a weibull mixtured distribution if its probability density function is defined as

$$
f(x ; a, b, \alpha)=\int_{0}^{\infty} a b r^{b-1} e^{-a r^{b}} g(x ; \alpha) d r
$$

Where $g(x, \alpha)$ is a probability density function. The name of weibull mixture distribution comes from the fact that the distribution (3.1) is the weighted average of $g(x, \alpha)$ with weights equal to the ordinates of weibull distribution.

\section{Definition 3.2}

If $\mathrm{X}$ follows a weibull mixture of Normal distribution with parameters $a$ and $b$, then the density function is given by

$$
f(x ; a, b)=\int_{0}^{\infty} a b r^{b-1} e^{-a r^{b}} \frac{e^{-\frac{1}{2} x^{2}} x^{2 r}}{2^{r+\frac{1}{2}} \sqrt{\left(r+\frac{1}{2}\right)}} d r ;-\infty<x<\infty
$$

with parameters $a$ and $b$ such that

$$
\int_{-\infty}^{\infty} f(x ; a, b) d x=1 .
$$

The characteristic function and moments of the same distribution are presented in the theorem below.

\section{Theorem 3.1}

If $\mathrm{X}$ has a weibull mixture of normal distributions with parameters $\mathrm{a}$ and $\mathrm{b}$ then its characteristic function is represented as

$$
\phi_{x}(t)=\int_{0}^{\infty} a b r^{b-1} e^{-a r^{b}} \frac{e^{-\frac{1}{t^{2}}}}{\left.2^{r+\frac{1}{2}}\right)\left(r+\frac{1}{2}\right)} \sum_{m=0}^{r}\left(\begin{array}{c}
2 r \\
2 m
\end{array}\right)(i t)^{2 m} 2^{r+\frac{1}{2}-m} \sqrt{\left(r+\frac{1}{2}-m\right)} d r(3.4)
$$

and the $2 s^{\text {th }}$ moment about origin is $\int_{0}^{\infty} a b r^{b-1} e^{-a r^{b}} 2^{s} \frac{\sqrt{\left(r+\frac{1}{2}+s\right)}}{\sqrt{\left(r+\frac{1}{2}\right)}} d r$

and $(2 s+1)^{\text {th }}$ moment about origin is zero. Mean $=0$,

$$
\text { Varinace }=1+2 a^{-\frac{1}{b}}\left(1+\frac{1}{b}\right), \beta_{1}=0, \beta_{2}=\frac{\left[3+8 a^{-\frac{1}{b}} \sqrt{\left(1+\frac{1}{b}\right)}+4 a^{-\frac{2}{b}} \sqrt{\left(1+\frac{2}{b}\right)}\right]}{\left[1+2 a^{-\frac{1}{b}}\left[\left(1+\frac{1}{b}\right)\right]^{2}\right.} .
$$

Remark: For $a=b=0, \phi_{x}(t), \mu_{2 s}^{\prime}, \mu_{(2 s+1)}^{\prime}, \mu_{1}, \mu_{2}, \mu_{3}, \mu_{4}, \beta_{1}$ and $\beta_{2}$ are same for Normal distribution with mean zero and variance unity.

*Corresponding author: Mian Arif Shams Adnan, Department Of Statistics, Jahangirnagar University, Savar, Dhaka 1342, Bangladesh, E-mail: julias284@yahoo.com

Received March 06, 2012; Accepted March 24, 2012; Published March 24, 2012

Citation: Adnan MAS, Kiser H (2012) A Class of Weibull Mixtured Distributions. J Biomet Biostat 3:135. doi:10.4172/2155-6180.1000135

Copyright: (c) 2012 Adnan MAS, et al. This is an open-access article distributed under the terms of the Creative Commons Attribution License, which permits unrestricted use, distribution, and reproduction in any medium, provided the original author and source are credited. 


\section{Definition 3.3}

If a random variable $\mathrm{X}$ has the density function

$$
f(x ; a, b)=\int_{0}^{\infty} a b r^{b-1} e^{-a r^{b}} \frac{e^{-\frac{1}{2}(\log x)^{2}}(\log x)^{2 r}}{\left.x 2^{r+\frac{1}{2}}\right)\left(r+\frac{1}{2}\right)} d r ; x>0
$$

then it is said to have a weibull mixture of Lognormal distribution with parameters $a, b$ since

$$
\int_{0}^{\infty} f(x ; a, b) d x=1
$$

Various moments of the distribution are given in the next theorem.

\section{Theorem 3.2}

If $X$ is a weibull mixture of lognormal variable with parameters $a, b$ then its characteristic function is given by

$$
\begin{aligned}
& \phi_{x}(t)=\int_{0}^{\infty} a b r^{b-1} e^{-a r^{b}} \frac{1}{\left.2^{r+\frac{1}{2}}\right)\left(r+\frac{1}{2}\right)} \sum_{k=0}^{\infty} \frac{(i t)^{k}}{k !} e^{\frac{1}{2} k^{2}} \\
& \sum_{m=0}^{r}\left(\begin{array}{c}
2 r \\
2 m
\end{array}\right) k^{2 r-2 m} 2^{m+\frac{1}{2}} \sqrt{\left(m+\frac{1}{2}\right)} d r
\end{aligned}
$$

and the $s^{\text {th }}$ moment about origin is

$$
\int_{0}^{\infty} a b r^{b-1} e^{-a r^{b}} \frac{e^{\frac{1}{s^{2}}}}{2^{r-m} \sqrt{\left(r+\frac{1}{2}\right)}} \sum_{m=0}^{r}\left(\begin{array}{c}
2 r \\
2 m
\end{array}\right) s^{2 r-2 m} \sqrt{\left(m+\frac{1}{2}\right)} d r .
$$

\section{Definition 3.4}

A random variable $\mathrm{X}$ having the density function

$$
f(x ; a, b, \alpha, \beta)=\int_{0}^{\infty} a b r^{b-1} e^{-a r^{b}} \frac{\beta^{\alpha+r} e^{-\beta x} x^{\alpha+r-1}}{\sqrt{(\alpha+r)}} d r ; x>0
$$

is defined a weibull mixture of Gamma distribution with parameters $a, b, \alpha$ and $\beta$ whereas

$$
\int_{0}^{\infty} f(x ; a, b, \alpha, \beta) d x=1 .
$$

The characteristic function and moments are followed by the next theorem.

\section{Theorem 3.3}

If $\mathrm{X}$ denotes a weibull mixture of gamma variate with parameters $a, b, \alpha$ and $\beta$ then its characteristic function is obtain as

$$
\begin{aligned}
& \phi_{x}(t)=a b\left(1-\frac{i t}{\beta}\right)^{-\alpha} \int_{0}^{\infty} r^{b-1} e^{-a r^{b}-r \ln \left(1-\frac{i t}{\beta}\right)} d r \\
& \text { Mean }=\frac{1}{\beta}\left[\alpha+a^{-\frac{1}{b}} \sqrt[\left(1+\frac{1}{b}\right)]{ }\right. \text {, } \\
& \text { Variance }=\frac{1}{\beta^{2}}\left[\alpha+a^{-\frac{1}{b}} \sqrt{\left(1+\frac{1}{b}\right)}+a^{-\frac{2}{b}} \sqrt{\left(1+\frac{2}{b}\right)}-\left\{a^{-\frac{1}{b}} \sqrt{\left(1+\frac{1}{b}\right)}\right\}^{2}\right] \text {, } \\
& \beta_{1}=\frac{\left[3 \left\{a^{-\frac{1}{b}} \sqrt{\left.\left.\left(1+\frac{1}{b}\right)\right\}\left\{a^{-\frac{2}{b}}\right)\left(1+\frac{2}{b}\right)\right\}+2\left\{a^{-\frac{1}{b}} \sqrt{\left(1+\frac{1}{b}\right)}+3 a^{-\frac{2}{b}} \sqrt{\left(1+\frac{2}{b}\right)}+a^{-\frac{3}{b}} \sqrt{\left(1+\frac{3}{b}\right)}-3\left\{a^{-\frac{1}{b}} \sqrt{\left(1+\frac{1}{b}\right)}\right\}^{2}-\right]^{2}}\right.\right.}{\left[\alpha+a^{-\frac{1}{b}} \sqrt{\left(1+\frac{1}{b}\right)}+a^{-\frac{2}{b}}\right)\left(1+\frac{2}{b}\right)-\left\{a^{-\frac{1}{b}} \sqrt{\left.\left.\left(1+\frac{1}{b}\right)\right\}^{2}\right]^{3}}\right.},
\end{aligned}
$$

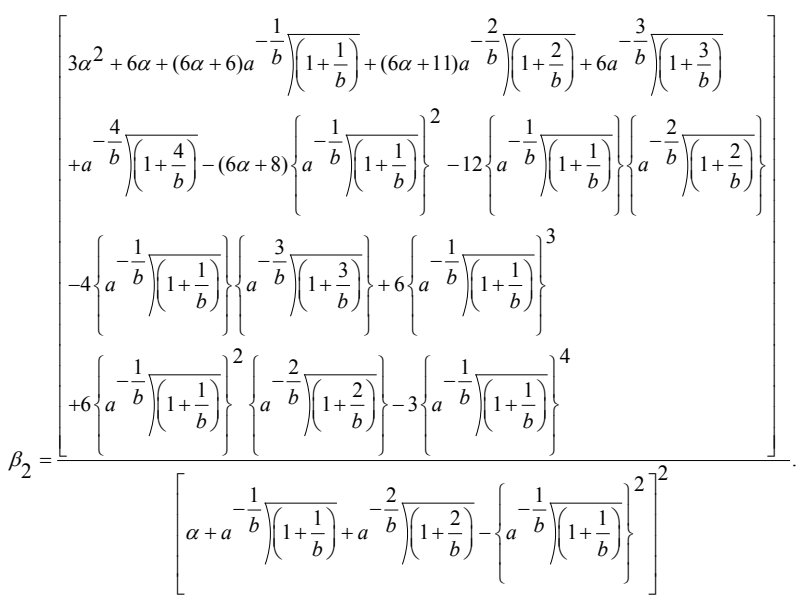

Remark: $\phi x(t), \mu_{1}, \mu_{2}, \mu_{3}, \mu_{1}, \beta_{1}$ and $\beta$ are true for Gamma distribution with parameters $\alpha$ and $\beta^{3}$ when $a=b^{2}=0$. For $\alpha=1$, weibull mixture of Gamma distribution should be equivalent to weibull mixture of Exponential distribution. As such we also derived the weibull mixture of Exponential distribution.

Estimates of parameters by the method of moments: Let $X_{1}, X_{2}$, $X_{3}, \ldots \ldots \ldots, X_{\mathrm{m}}$ be a random sample from the distribution (3.8). We assume that parameters $a, b$ and $\beta$ are known. Then the distribution contains only one unknown parameter $\alpha$. We have $\mu_{1}^{\prime}=\frac{1}{\beta}\left[\alpha+a^{-\frac{1}{b}} \sqrt{\left(1+\frac{1}{b}\right)}\right]$, and $m_{1}^{\prime}=\frac{\sum x_{i}}{m}=\bar{X}$. Hence by the method of moments, we get, $\frac{1}{\beta}\left[\alpha+a^{-\frac{1}{b}} \sqrt{\left(1+\frac{1}{b}\right)}\right]=\bar{X}$. Therefore, $\hat{\alpha}=\bar{X} \beta-a^{-\frac{1}{b}} \sqrt{\left(1+\frac{1}{b}\right)}$.

\section{Definition 3.5}

A random variable $\mathrm{X}$ having the density function

$$
f(x ; a, b, \alpha)=\int_{0}^{\infty} a b r^{b-1} e^{-a r^{b}} \frac{\alpha^{r+1} e^{-\alpha x} x^{r}}{\sqrt{(r+1)}} d r ; x>0
$$

is said to have a weibull mixture of Exponential distribution with parameters $a, b \alpha$, and

$$
\int_{0}^{\infty} f(x ; a, b, \alpha) d x=1
$$

\section{Theorem 3.4}

If $X$ follows weibull mixture of exponential distributions with parameters $a, b$ and $\alpha$ then its characteristic function is given by

$$
\begin{aligned}
& \phi_{x}(t)=a b\left(1-\frac{i t}{\alpha}\right)^{-1} \int_{0}^{\infty} r^{b-1} e^{-a r^{b}-r \ln \left(1-\frac{i t}{\alpha}\right)} d r, \\
& \text { Mean }=\frac{1}{\alpha}\left[1+a^{-\frac{1}{b}} \overline{\left(1+\frac{1}{b}\right)}\right] \text {, } \\
& \text { Variance }=\frac{1}{\alpha^{2}}\left[1+a^{-\frac{1}{b}} \sqrt{\left(1+\frac{1}{b}\right)}+a^{-\frac{2}{b}} \sqrt{\left(1+\frac{2}{b}\right)}-\left\{a^{-\frac{1}{b}} \sqrt{\left(1+\frac{1}{b}\right)}\right\}^{2}\right] \text {, } \\
& \beta_{1}=\frac{\left[-3\left\{a^{-\frac{1}{b}} \sqrt{\left.\left(1+\frac{1}{b}\right)\right\}\left\{a^{-\frac{2}{b}} \sqrt{\left(1+\frac{1}{b}\right)}\right\}+2\left\{a^{-\frac{1}{b}} \sqrt{\left(1+\frac{1}{b}\right)}+3 a^{-\frac{2}{b}} \sqrt{\left(1+\frac{2}{b}\right)}+a^{-\frac{3}{b}} \sqrt{\left(1+\frac{3}{b}\right)}-3\left\{a^{-\frac{1}{b}} \sqrt{\left(1+\frac{1}{b}\right)}\right\}^{2}\right]^{2}}\right.\right.}{\left[1+a^{-\frac{1}{b}} \sqrt{\left(1+\frac{1}{b}\right)}+a^{-\frac{2}{b}} \sqrt{\left(1+\frac{2}{b}\right)}-\left\{a^{-\frac{1}{b}} \sqrt{\left(1+\frac{1}{b}\right)}\right\}^{2}\right]^{3}},
\end{aligned}
$$




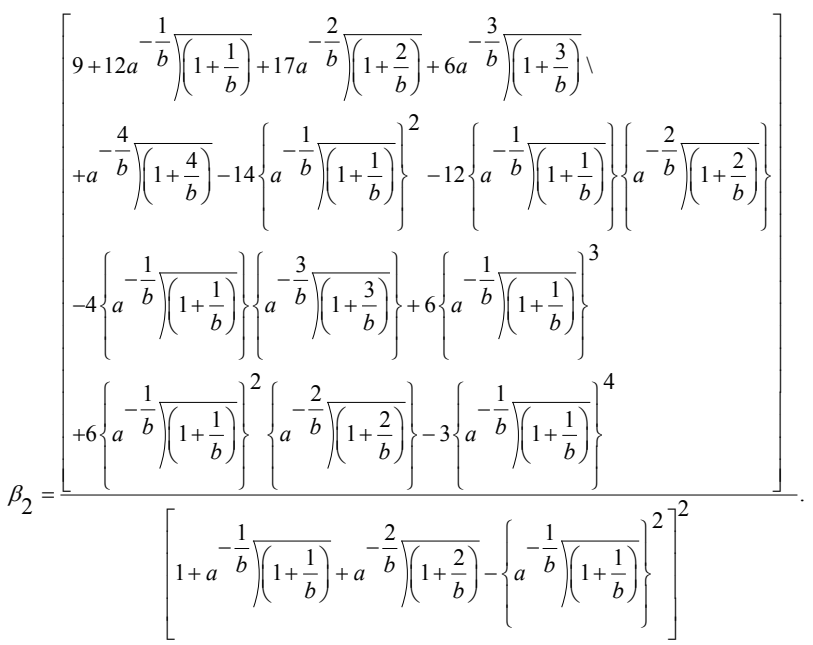

Remark: When $a=b=0$, then all of $\Pi_{x}(t), \mu_{1}, \mu_{2}, \mu_{3}, \mu_{4}, \beta_{1}$ and $\beta_{2}$ are similar to these of Exponential distribution with parameter $\alpha$.

Parameter estimation: If $X_{1}, X_{2}, X_{3}, \ldots \ldots ., X_{m}$ be a random sample drawn from the distribution (3.12) and parameters a,b are assumed known, then the distribution contains only one unknown parameter $\alpha$. So, $\mu_{1}^{\prime}=\frac{1}{\alpha}\left[1+a^{-\frac{1}{b}} \sqrt{\left(1+\frac{1}{b}\right)}\right]$, and $m_{1}^{\prime}=\frac{\sum x_{i}}{m}=\bar{X}$. Therefore, $\frac{1}{\alpha}\left[1+a^{-\frac{1}{b}} \sqrt{\left(1+\frac{1}{b}\right)}\right]=\bar{X}$. Hence, $\hat{\alpha}=\frac{\left[1+a^{-\frac{1}{b}} \overline{\left(1+\frac{1}{b}\right)}\right]}{\bar{X}}$

\section{Definition 3.6}

If a random variable $\mathrm{X}$ has the density function

$$
f(x ; a, b, \alpha, \beta)=\int_{0}^{\infty} a b r^{b-1} e^{-a r^{b}} \frac{(\alpha \beta)^{\alpha+r} e^{-\alpha \beta x} x^{\alpha+r-1}}{\sqrt{(\alpha+r)}} d r ; x>0
$$

then it is said to have a weibull mixture of Erlang distribution with parameters $\mathrm{a}, \mathrm{b}, \alpha$ and $\beta$ since

$$
\int_{0}^{\infty} f(x ; a, b, \alpha, \beta) d x=1
$$

The characteristic function as well as the moments is stated in the following theorem.

\section{Theorem 3.5}

If $X$ has weibull mixture of erlang distributions with parameters $a, b, \alpha$ and $\beta$ then its characteristic function is given by

$$
\begin{aligned}
& \phi_{x}(t)=a b\left(1-\frac{i t}{\alpha \beta}\right)^{-\alpha} \int_{0}^{\infty} r^{b-1} e^{-a r^{b}-r \ln \left(1-\frac{i t}{\alpha \beta}\right)} d r \\
& \text { Mean }=\frac{1}{\alpha \beta}\left[\alpha+a^{-\frac{1}{b}} \overline{\left(1+\frac{1}{b}\right)}\right] \text {, } \\
& \text { Variance }=\frac{1}{(\alpha \beta)^{2}}\left[\alpha+a^{-\frac{1}{b}} \sqrt{\left(1+\frac{1}{b}\right)}+a^{-\frac{2}{b}} \sqrt{\left(1+\frac{2}{b}\right)}-\left\{a^{-\frac{1}{b}} \sqrt[\left(1+\frac{1}{b}\right)]{ }\right\}^{2}\right] \text {, } \\
& \beta_{1}=\frac{\left[-3\left\{a^{-\frac{1}{b}} \sqrt{\left.\left(1+\frac{1}{b}\right)\right\}\left\{a^{-\frac{2}{b}} \sqrt{\left(1+\frac{1}{b}\right)}\right\}+2\left\{a^{-\frac{1}{b}} \sqrt{\left.\left(1+\frac{1}{b}\right)\right\}^{3}}+3 a^{-\frac{2}{b}} \sqrt{\left(1+\frac{2}{b}\right)}+a^{-\frac{3}{b}} \sqrt{\left(1+\frac{3}{b}\right)}-3\left\{a^{-\frac{1}{b}} \sqrt{\left(1+\frac{1}{b}\right)}\right\}^{2}\right]^{2}}\right.\right.}{\left[\alpha+a^{-\frac{1}{b}} \sqrt{\left(1+\frac{1}{b}\right)}+a^{-\frac{2}{b}} \sqrt{\left(1+\frac{2}{b}\right)}-\left\{a^{-\frac{1}{b}} \sqrt{\left(1+\frac{1}{b}\right)}\right\}^{2}\right]^{3}},
\end{aligned}
$$

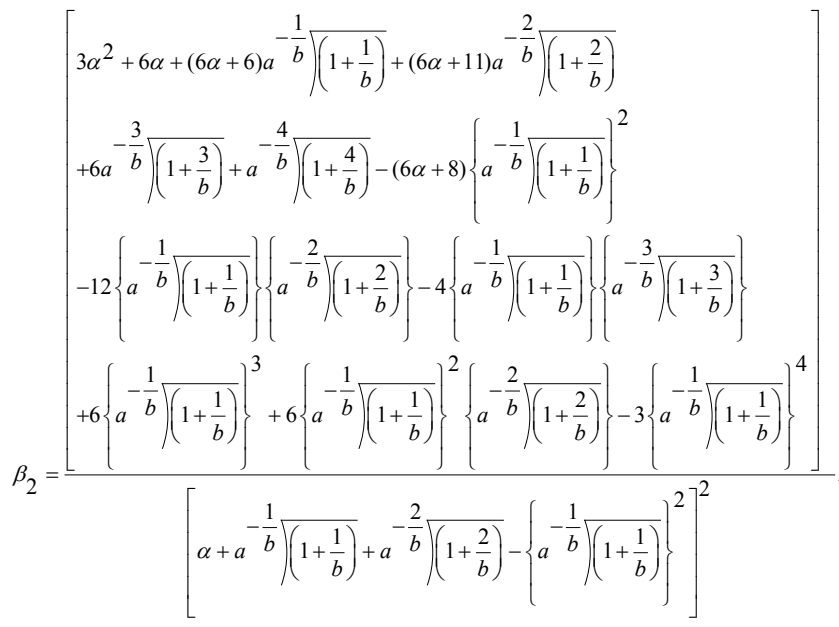

Remark: $a=b=0$ provides all the values of $\phi x(\mathrm{t}), \mu_{1}, \mu_{2}, \mu_{3}, \mu_{4}, \beta_{1}$ and $\beta_{2}$ to be true for Erlang distribution with parameters $\alpha$ and $\beta$.

Estimating parameters: For a random sample $X_{1}, X_{2}, X_{3}, \ldots \ldots \ldots, X_{\mathrm{m}}$ from the distribution (3.16), we assume that parameters $a, b$ and $\beta$ are known and $\alpha$ unknown parameter. Here, $\mu_{1}^{\prime}=\frac{1}{\alpha \beta}\left[\alpha+a^{-\frac{1}{b}}\left(1+\frac{1}{b}\right)\right]$, and $m_{1}^{\prime}=\frac{\sum x_{i}}{m}=\bar{X}$. We obtain $\frac{1}{\alpha \beta}\left[\alpha+a^{-\frac{1}{b}} \sqrt[\left(1+\frac{1}{b}\right)]{ }\right]=\bar{X}$. Therefore,

$$
\hat{\alpha}=\frac{a^{-\frac{1}{b}} \sqrt{\left(1+\frac{1}{b}\right)}}{(\bar{X} \beta-1)}
$$

\section{Definition 3.7}

A random variable $\mathrm{X}$ having the density function

$$
f(x ; a, b, m)=\int_{0}^{\infty} a b r^{b-1} e^{-a r^{b}} \frac{(r+1) x^{r}}{m^{r+1}} d r ; 0<x<m
$$

is said as weibull mixture of Rectangular distribution with parameters $a, b$ and $m$ satisfying

$\int_{0}^{m} f(x ; a, b, m) d x=1$.

Different moments of the above mentioned distribution are expressed below.

\section{Theorem 3.6}

If $X$ follows a weibull mixture of rectangular distribution with parameters $a, b$ and $m$ then its characteristic function is obtained as

$$
\phi_{x}(t)=\int_{0}^{\infty} a b r^{b-1} e^{-a r^{b}} \sum_{k=0}^{\infty} \frac{(i t)^{k}(r+1) m^{r+k+1}}{k ! m^{r+1}(r+k+1)} d r
$$

and the $s^{\text {th }}$ moment about origin is $m^{s} \int_{0}^{\infty} a b r^{b-1} e^{-a r^{b}} \frac{r+1}{r+s+1} d r$.

Remark: If $a=b=0$ then all the values of $\phi_{x}(t), \mu_{1}, \mu_{2}, \mu_{3}, \mu_{4}, \beta_{1}$ are true for Rectangular distribution with parameter $m$.

\section{Definition 3.8}

A random variable $\mathrm{X}$ having the density function

$$
f(x ; a, b, \alpha, \beta)=\int_{0}^{\infty} a b r^{b-1} e^{-a r^{b}} \frac{x^{\alpha+r-1}(1-x)^{\beta-1}}{B(\alpha+r, \beta)} d r ; 0<x<1
$$

is said to have a weibull mixture of Beta distribution of $1^{\text {st }}$ kind with parameters $a, b, \alpha$ and $\beta$. Here we have 


$$
\int_{0}^{1} f(x ; a, b, \alpha, \beta) d x=1
$$

\section{Theorem 3.7}

If $\mathrm{X}$ follows weibull mixture of beta distributions of first kind with parameters $a, b, \alpha$ and $\beta$, then its $s^{\text {th }}$ moment about origin is given by

$$
\phi_{x}(t)=\int_{0}^{\infty} a b r^{b-1} e^{-a r^{b}} \frac{B(\alpha+s+r, \beta)}{B(\alpha+r, \beta)} d r
$$

Remark: For $a=b=0$, all the values of $\mu_{s}^{\prime}, \mu_{1}^{\prime}, \mu_{2}^{\prime}$ and $\mu_{2}$ are true for Beta distribution of $1^{\text {st }}$ kind with parameters $\alpha$ and $\beta$.

\section{Definition 3.9}

A random variable $\mathrm{X}$ having the density function

$$
f(x ; a, b, \alpha, \beta)=\int_{0}^{\infty} a b r^{b-1} e^{-a r^{b}} \frac{x^{\alpha+r-1}}{B(\alpha+r, \beta)(1+x)^{\alpha+\beta+r}} d r ; x>0
$$

is called a weibull mixture of Beta distribution of $2^{\text {nd }}$ kind with parameters $a, b, \alpha$ and $\beta$. Moreover,

$$
\int_{0}^{\infty} f(x ; a, b, \alpha, \beta) d x=1
$$

Next theorem presents some properties of the same distribution.

\section{Theorem 3.8}

If $X$ follows weibull mixture of beta distribution of second kind with parameters $a, b, \alpha$ and $\beta$ then its $s^{\text {th }}$ moment about origin is given by $\int_{0}^{\infty} a b r^{b-1} e^{-a r^{b}} \frac{B(\alpha+s+r, \beta-s)}{B(\alpha+r, \beta)} d r$.

Remark: Putting $a=b=0$ then all the values of $\mu_{s}^{\prime}, \mu_{1}^{\prime}, \mu_{2}^{\prime}$ and $\mu_{2}$ are true for Beta distribution of $2^{\text {nd }}$ kind with parameters $\alpha$ and $\beta$.

\section{Definition 3.10}

A random variable $X^{2}$ with the density function

$$
f\left(\chi^{2} ; a, b, n\right)=\int_{0}^{\infty} a b r^{b-1} e^{-a r^{b}} \frac{e^{-\frac{1}{2} \chi^{2}}\left(\chi^{2}\right)^{\frac{n}{2}+r-1}}{2^{\frac{n}{2}+r} \sqrt{\frac{n}{2}+r}} d r ; \chi^{2}>0
$$

is said to have a weibull mixture of Chi-square distribution having the parameters $a, b$ and $n$ since

$$
\int_{0}^{\infty} f\left(\chi^{2} ; a, b, n\right) d \chi^{2}=1 .
$$

\section{Theorem 3.9}

If $X^{2}$ has weibull mixture chi-square distribution with parameters $a, b$ and $n$ then its characteristic function is expressed as

$$
\begin{aligned}
& \phi_{x}(t)=a b(1-2 i t)^{-\frac{n}{2}} \int_{0}^{\infty} a b r^{b-1} e^{-a r^{b}-r \ln (1-2 i t)} d r \\
& \text { Mean }=n+2 a^{-\frac{1}{b}} \sqrt{\left(1+\frac{1}{b}\right)},
\end{aligned}
$$

\begin{tabular}{|c|c|c|c|c|}
\hline SI. & Name of the distribution & Probability density function $f(x)$ & Support & Parameters \\
\hline 1 & Weibull mixtured Normal & $\int_{0}^{\infty} a b r^{b-1} e^{-a a^{r}} \frac{e^{-\frac{1}{2} x^{2}} x^{2 r}}{2^{r+\frac{1}{2}}\left(\sqrt{\left(r+\frac{1}{2}\right)}\right.} d r$ & $-\infty<x<\infty$ & $a, b$ \\
\hline 2 & Weibull mixtured Lognormal & 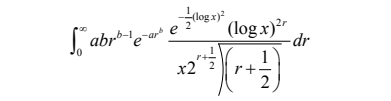 & $x>0$ & $a, b$ \\
\hline 3 & Weibull mixtured Gamma & $\int_{0}^{\infty} a b r^{b-1} e^{-a r^{\phi}} \frac{\beta^{\alpha+r} e^{-\beta \gamma} x^{\alpha+r-1}}{\sqrt{(\alpha+r)}} d r$ & $x>0$ & $a, b, \alpha, \beta$ \\
\hline 4 & Weibull mixtured Exponential & $\int_{0}^{\infty} a b r^{b-1} e^{-a r^{r}} \frac{\alpha^{r+1} e^{-\alpha x} x^{r}}{\sqrt{(r+1)}} d r$ & $x>0$ & $a, b, a$ \\
\hline 5 & Weibull mixtured Erlang & $\int_{0}^{\infty} a b r^{b-1} e^{-a r^{\beta}} \frac{(\alpha \beta)^{\alpha+r} e^{-\alpha \beta \gamma} x^{\alpha+r-1}}{\sqrt{(\alpha+r)}} d r$ & $x>0$ & $a, b, \alpha, \beta$ \\
\hline 6 & Weibull mixtured Rectangular & $\int_{0}^{\infty} a b r^{b-1} e^{-a r^{b}} \frac{(r+1) x^{r}}{m^{r+1}} d r$ & $0<x<m$ & $a, b, m$ \\
\hline 7 & Weibull mixtured Beta $1^{\text {st }}$ kind & $\int_{0}^{\infty} a b r^{b-1} e^{-a r^{\beta}} \frac{x^{\alpha+r-1}(1-x)^{\beta-1}}{B(\alpha+r, \beta)} d r$ & $0<x<1$ & $a, b, \alpha, \beta$ \\
\hline 8 & Weibull mixtured Beta $2^{\text {nd }}$ kind & $\int_{0}^{\infty} a b r^{b-1} e^{-a r^{\beta}} \frac{x^{\alpha+\gamma-1}}{B(\alpha+r, \beta)(1+x)^{\alpha+\beta+r}} d r$ & $x>0$ & $a, b, \alpha, \beta$ \\
\hline 9 & Weibull mixtured Chi-square & $\int_{0}^{\infty} a b r^{b-1} e^{-a r^{b}} \frac{e^{-\frac{1}{2} x^{2}}\left(\chi^{2}\right)^{\left.\frac{n}{2}\right)^{\frac{n}{2}-1}}}{\left.2^{\frac{n}{2}+r}\right) \frac{n}{2}+r} d r$ & $\chi^{2}>0$ & $a, b, n$ \\
\hline 10 & Weibull mixtured $t$ & $\int_{0}^{\infty} a b r^{b-1} e^{-a^{\beta}} \frac{t^{2 r}}{n^{\frac{1}{2}+r} B\left(\frac{1}{2}+r, \frac{n}{2}\right)\left(1+\frac{t^{2}}{n}\right)^{\frac{n+1}{2}+r}} d r$ & $-\infty<t<\infty$ & $a, b, n$ \\
\hline 11 & Weibull mixtured $F$ & $\int_{0}^{\infty} a b r^{b-1} e^{-a r^{b}} \frac{\left(\frac{n_{1}}{n_{2}}\right)^{\frac{n_{4}+r}{2}} F^{\frac{n_{1}+r-1}{2}}}{B\left(\frac{n_{1}}{2}+r, \frac{n_{2}}{2}\right)\left(1+\frac{n_{1}}{n_{2}} F\right)^{\frac{n_{1}+n_{2}}{2}+r}} d r$ & $F>0$ & $a, b, n^{1}, n^{2}$ \\
\hline
\end{tabular}

Remark: Setting $\mathrm{a}=\mathrm{b}=0$ we find that all the values of $\phi_{x}(t), \mu_{1}, \mu_{2}, \mu_{3}, \mu_{4}, \beta_{1}$ and $\beta_{2}$ are true for Chi-square distribution with parameters $n$.

Parameter estimation: Let $X_{1}, X_{2}, X_{3}, \ldots \ldots \ldots, X_{m}$ be a random sample from the distribution (3.28). We assume that parameters $a$ and $b$ are known and $\mathrm{n}$ is unknown. Now, $\mu_{1}^{\prime}=n+2 a^{-\frac{1}{b}} \sqrt{\left(1+\frac{1}{b}\right)}$, and $m_{1}^{\prime}=\frac{\sum x_{i}}{m}=\bar{X}$. As such, $n+2 a^{-\frac{1}{b}} \sqrt{\left(1+\frac{1}{b}\right)}=\bar{X}$. Therefore, $\hat{n}=\bar{X}-2 a^{-\frac{1}{b}} \sqrt{\left(1+\frac{1}{b}\right)}$ (3.31)

Table 1: Comparison of density functions of different Weibull mixture distributions. $\chi^{2}>0$. 


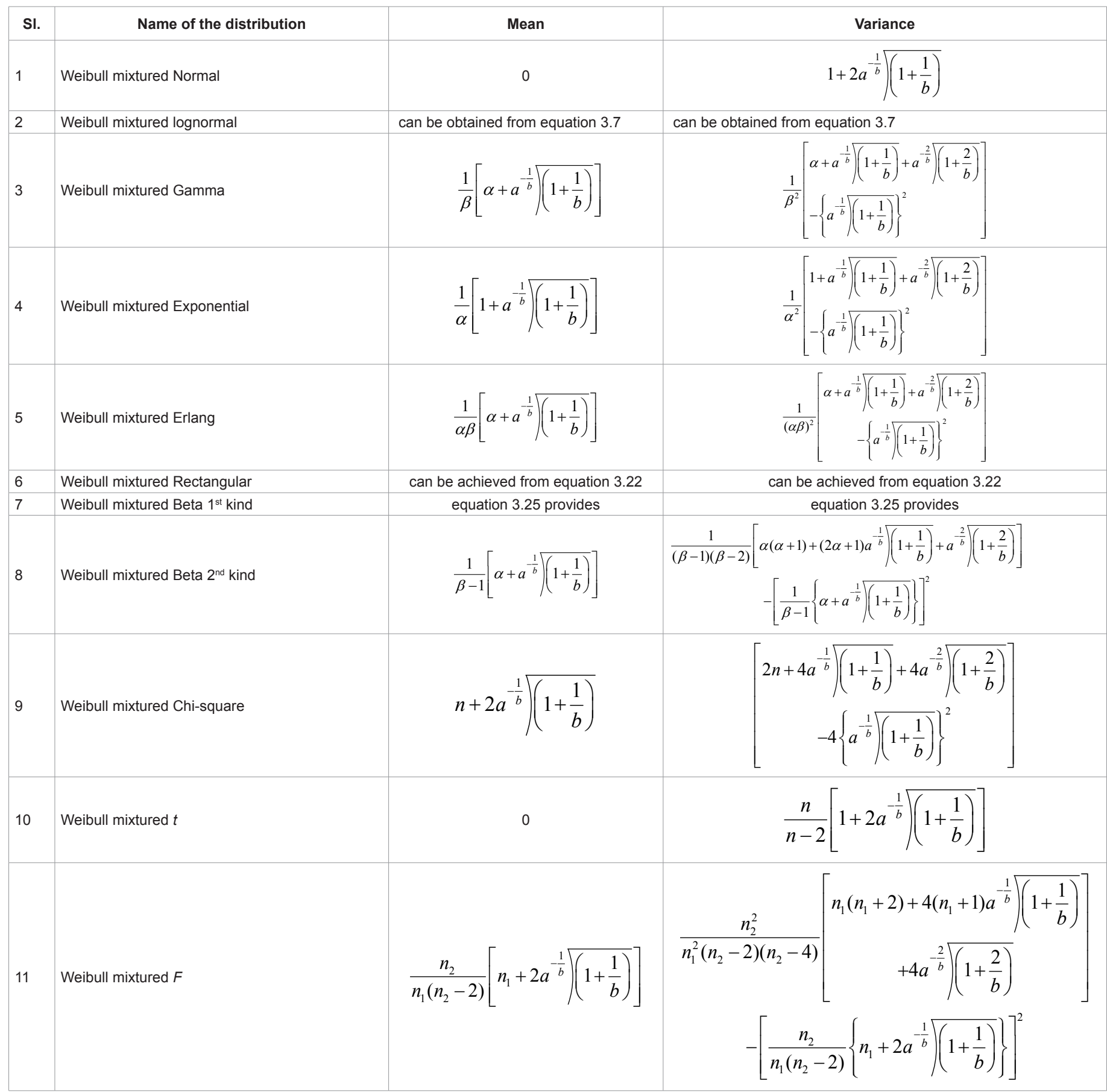

\section{Definition 3.11}

If $t$ as a random variable has the density function

$$
f(t ; a, b, n)=\int_{0}^{\infty} a b r^{b-1} e^{-a r^{b}} \frac{t^{2 r}}{n^{\frac{1}{2}+r} B\left(\frac{1}{2}+r, \frac{n}{2}\right)\left(1+\frac{t^{2}}{n}\right)^{\frac{n+1}{2}+r}} d r ;-\infty<t<\infty
$$

then it is said to have a weibull mixture of $t$ distribution with parameters $a, b$ and $n$ if

$$
\int_{-\infty}^{\infty} f(t ; a, b, n) d t=1
$$

The following theorem expresses here some of the properties of the distribution.

\section{Theorem 3.10}

If $\mathrm{t}$ is weibull mixture of $\mathrm{t}$ distribution with parameters $a, b$ and $n$ then the $2 s^{\text {th }}$ moment about origin is given by $n^{s} \int_{0}^{\infty} a b r^{b-1} e^{-a r^{b}} \frac{\sqrt{\left.\left(r+s+\frac{1}{2}\right)\right)\left(\frac{n}{2}-s\right)}}{\sqrt{\left(\frac{1}{2}+r\right)}} d r$ and the $(2 s+1)^{t h}$ moment about origin 
is zero, $\beta_{1}=0, \beta_{2}=\frac{n-2}{n-4} \frac{\left[3+8 a^{-\frac{1}{b}} \sqrt{\left(1+\frac{1}{b}\right)}+4 a^{-\frac{2}{b}} \sqrt{\left(1+\frac{2}{b}\right)}\right]}{\left.\left[1+2 a^{-\frac{1}{b}}\right)\left(1+\frac{1}{b}\right)\right]^{2}}$.

Remark: If $a=b=0$ then all the values of $\mu_{2 s+1}, \mu_{2 s}, \mu_{1}, \mu_{2}, \mu_{3}, \mu_{4}, \beta_{1}$ and $\beta_{2}$ are true for $t$ distribution with parameter $n$.

\section{Definition 3.12}

A random variable $F$ having the density function

$$
f\left(F ; a, b, n_{1}, n_{2}\right)=\int_{0}^{\infty} a b r^{b-1} e^{-a r^{b}} \frac{\left(\frac{n_{1}}{n_{2}}\right)^{\frac{n_{1}+r}{2}} F^{\frac{n_{1}}{2}+r-1}}{B\left(\frac{n_{1}}{2}+r, \frac{n_{2}}{2}\right)\left(1+\frac{n_{1}}{n_{2}} F\right)^{\frac{n_{1}+n_{2}}{2}+r}} d r ; F>0
$$

is said to have a weibull mixture of $F$ distribution with parameters $a, b$, $n_{1}$ and $n_{2}$ if

$$
\int_{0}^{\infty} f\left(F ; a, b, n_{1}, n_{2}\right) d F=1
$$

The following theorem presents the characteristic function and moments of this distribution.

\section{Theorem 3.11}

If $F$ follows weibull mixture of $F$ distribution with parameters $a, b$, $n_{1}$ and $n_{2}$ then its characteristic function is given by

$$
\phi_{x}(t)=\int_{0}^{\infty} a b r^{b-1} e^{-a r^{b}} \sum_{x=0}^{\infty} \frac{\left(i t \frac{n_{2}}{n_{1}}\right)^{x}}{x !} \frac{\sqrt{\left.\left(\frac{n_{2}}{2}+r+x\right)\right) \sqrt{\left(\frac{n_{2}}{2}-x\right)}}}{\sqrt{\left.\left(\frac{n_{1}}{2}+r\right)\right)\left(\frac{n_{2}}{2}\right)}} d r
$$

and the $S^{\text {th }}$ momentaboutorigin is $\left(\frac{n_{2}}{n_{1}}\right)^{s} \int_{0}^{\infty} a b r^{b-1} e^{-a r^{b}} \frac{\sqrt{\left(\frac{n_{2}}{2}+r+s\right) \sqrt{\left(\frac{n_{2}}{2}-s\right)}}}{\sqrt{\left.\left(\frac{n_{1}}{2}+r\right)\right)\left(\frac{n_{2}}{2}\right)}} d r$,
Remark: For $a=b=0$ all the values of $\phi_{x}(t), \mu_{1}, \mu_{2}$ and $\mu_{2}$ are true for $F$ distribution with parameters $n_{1}$ and $n_{2}$.

\section{Comparison}

A Comparison among various features of the different weibull mixtured distributions is shown in the following table 1 and table 2.

\section{References}

1. Pearson K (1894) Contributions To The Mathematical Theory of Evolution. Philos Trans R Soc London 185: 71-110.

2. Rider PR (1961) The Method of Moments Applied To A Mixture of Two Exponential Distributions. The Annals of Mathematical Statistics 32: 143-147.

3. Blichke WR (1965) Mixtures of Discrete Distribution. Classical And Contagious Discrete Distributions. Calcutta Statistical Publishing Society. Ed GP Patil 351 372.

4. Chahine J (1965) Une Generalization Dela Loi Binomiale Negative. Rev Stat Appl 13: 33-43.

5. Roy MK, Rahman S, Ali MM (1992) A Class Of Poisson Mixtured Distributions J Info And Optimiz Sci 13: 207-208.

6. Roy MK (1998) Some Negative Binomial Mixtured Distributions. J Info And Optimiz Sci 19: 285-302.

7. Roy MK, Sinha SK (1995) Negative Binomial Mixture of Normal Moment Distributions. Metron 3: 83-92.

8. Authors (2009) Some Laplace Mixture Distributions. J Appli Stati Sci 17: 549 560 .

9. Authors (2010) Some Pareto Mixtured Distributions. Proceedings. American Statistical Association.

10. Authors (2011) A Class of Rayleigh Mixture Distributions. Journal of Interdisciplinary Mathematics 14: 507-522.

11. Author (2011) A Mixture Probabilistic Model for Extreme Distributions. Proceedings. American Statistical Association.

12. Authors (2012) A Class of Mixture Distributions. Journal of Interdisciplinary Mathematics. To appear.

13. Authors (2012) A Class of Dual Mixture Distributions. Journal of Interdisciplinary Mathematics. To appear.

14. Johnson NL, Kotz S, Kemp AW (1992) Univariate Discrete Distributions. (2 Edition) Wiley, New York.

15. Johnson NL, Kotz S, Balakrishnan N (1995) Continuous Univariate Distributions ( $2^{\text {nd }}$ Edition) Wiley, New York. 